\title{
SOBRE EL POTENCIAL CRÍTICO Y EMANCIPADOR DE LA HERMENÉUTICA GADAMERIANA DESDE LA PERSPECTIVA DE LOS ESTUDIOS LITERARIOS*
}

\author{
Hanna MERETOJA \\ Universidad de Turku
}

\begin{abstract}
RESUMEN. En este artículo me enfrento a la concepción según la cual la hermenéutica filosófica representa una tradición intelectual intrínsecamente acrítica o conservadora, y examino su relevancia para la autocomprensión teórica de los estudios literarios. Argumento que tal relevancia no puede apreciarse en su totalidad desde una perspectiva meramente metodológica. Partiendo de la dimensión ontológico-trascendental de la hermenéutica gadameriana, explico cuál es el potencial crítico que se abre gracias a sus tesis sobre la naturaleza fundamentalmente histórica y dialógica de la comprensión. De ahí que los estudios literarios puedan concebirse como un proceso dialógico que lanza un reto en una doble dirección: tanto hacia el mundo histórico perteneciente al texto como hacia el perteneciente al intérprete. Semejante práctica de cuestionamiento crítico hace patente la historicidad de los sistemas significativos existentes, al mismo tiempo que articula nuevas posibilidades abiertas precisamente por parte de los textos literarios.
\end{abstract}

Sigue siendo una opinión común entre los estudiosos provenientes de diversos campos humanísticos la noción de que la hermenéutica gadameriana representa un acercamiento esencialmente conservador y tradicionalista a las ciencias humanas. Esta visión se ha debido en gran parte a la manera en que la hermenéutica de Gadamer se ha opuesto a la teoría crítica tras su debate con Jürgen

\footnotetext{
* Traducción del inglés por Gorka Fernández Villarón.
} 
Habermas sobre la pretensión de universalidad de la hermenéutica. Los malentendidos que definieron esta discusión han dominado obstinadamente la recepción de la hermenéutica. En este debate, el juicio de Habermas sobre la insuficiencia de la hermenéutica se fundó en una perspectiva predominantemente metodológica: sostuvo que el mero "comprender" no puede proporcionar una base metodológica suficiente para la ciencia social crítica y debe suplirse por procedimientos explicativos. ${ }^{1}$ Para Gadamer, sin embargo, la pretensión de universalidad de la hermenéutica nunca fue una pretensión metodológica sino más bien una pretensión que se hacía desde un punto de vista ontológico-trascendental: según tal pretensión, la comprensión era la manera humana básica de estar en el mundo, y por tanto la hermenéutica, es decir, la filosofía que aclara la naturaleza y las condiciones del comprender, se referiría a todos los aspectos de la existencia humana, incluyendo todas las formas de experiencia y de conocimiento así como todas las ciencias. En Verdad y método, Gadamer se preguntó por las condiciones de posibilidad de las ciencias humanas desde este punto de partida universal. ${ }^{2}$

Más tarde Habermas abandonaría la oposición entre comprensión y explicación, en gran parte debido al giro postempirista de la filosofía analítica angloamericana de la ciencia, que había cuestionado esta dicotomía al demostrar que también las ciencias naturales practican la interpretación de la realidad con sus

1 Desafortunadamente, no puedo entrar aquí en los detalles de este debate. Para las contribuciones más importantes de Habermas al debate, véase HABERMAS 1971; HABERMAS 1986a. Aunque él ha revisado más adelante sus opiniones sobre la hermenéutica de Gadamer, no ha abandonado su perspectiva metodológica. Por ejemplo, en su teoría de la acción comunicativa hace una lista de seis puntos a propósito de "la contribución metodológica de la hermenéutica filosófica" (HABERMAS 1984, 135).

2 Véase por ejemplo GW2, 238-240, 438-440; TM 259-264, 474-476/GW, 264-268, 478480. Como Gadamer aclara, su Verdad y método no trata de decir cómo deben ser las ciencias humanas, sino que se pregunta por su "posibilidad trascendental": «En el sentido de esta distinción kantiana, pensar más allá del concepto del método en las ciencias humanas, como mi libro procura, es plantearse la cuestión de la "posibilidad" de las ciencias humanas (que no significa ciertamente lo que realmente deberían ser)" (TM 512/GW2, 394). Sobre la dimensión trascendental de la filosofía hermenéutica, véase BUBNER 1973, 89-111; DOSTAL 2002, 252; SASTRE 1995, 20-33. Está claro que la hermenéutica gadameriana se diferencia de manera decisiva de la filosofía trascendental kantiana o husserliana, pues Gadamer, siguiendo a Heidegger, abandona el sujeto trascendental y la búsqueda concomitante de la certeza inmediata o la autotrasparencia absoluta del sujeto trascendental, pero replantea, sin embargo, la pregunta trascendental sobre las condiciones de posibilidad de la experiencia humana del mundo. 
esquemas conceptuales. ${ }^{3}$ Este giro ha incrementado indudablemente la popularidad de la hermenéutica y el reconocimiento de su importancia general para la filosofía de la ciencia: muchos filósofos americanos hablan del "giro interpretativo" gracias al cual la filosofía analítica se ha acercado a la hermenéutica, mientras que en Europa Gianni Vattimo ha llegado a reputar a la hermenéutica como la nueva koiné de la filosofía y de la cultura contemporáneas en general. ${ }^{4}$ Este desarrollo, sin embargo, no ha supuesto ningún cambio significativo en la actitud general hacia la hermenéutica gadameriana como algo acrítico y conservador. ${ }^{5}$ Por supuesto, hay especialistas, como G. B. Madison, que han sostenido constantemente que la hermenéutica gadameriana promueve el ejercicio de la razón crítica, pero tales opiniones parecen no haber obtenido hasta ahora mucho reconocimiento, o por lo menos no entre la mayoría de los círculos académicos. ${ }^{6}$

Como Gadamer ha indicado, su debate con Habermas se refirió esencialmente a la cuestión de si la hermenéutica puede o no «responder a la verdadera necesidad de este tiempo, es decir, la emancipación de viejas tradiciones y convenciones heredadas" y "proporcionar lo más importante ahora, a saber, crítica». ${ }^{7}$ Gadamer estaba convencido de que puede: para él, su hermenéutica filosófica corresponde a "lo que Kant denomina Ilustración", es decir, "la práctica de la facultad de juicio». ${ }^{8}$ Como sabemos, Kant se refería con «Ilustración» al valor de pensar por uno mismo, rechazando de tal modo "la minoría de edad autoimpuesta». ${ }^{9}$ En este ar-

3 Véase Habermas 1984, 109-110. Habermas se refiere con «el giro postempirista de la teoría analítica de la ciencia" al trabajo de Mary Hesse, Thomas Kuhn, Karl Popper, Imre Lakatos, y Paul Feyerabend, y según él, resulta crucial en este giro darse cuenta de que «los datos con los que se prueban las teorías no pueden ser descritos independientemente de la teoría del lenguaje en cuestión", y que "las teorías están construidas [...] dentro de ciertos paradigmas» (ibíd., 109). Cf. GIDDENS 1976, 68.

4 VATTIMO 1988, 399. Sobre "el giro interpretativo", véase BOHMAN et al. 1991, 1-4. Este giro ha llevado a, por ejemplo, rechazar las suposiciones referentes a "la neutralidad de la observación, lo 'dado' de la experiencia, la independencia de los datos empíricos respecto de los marcos teóricos" (ibíd., p. 3). Cf. Bernstein 1985, 30-33.

5 Habermas, por ejemplo, repite sus opiniones sobre Gadamer como alguien "conservador" en sus últimos escritos (véase HABERMAS 2001, 91).

6 MADISON 2001, 27.

7 Gadamer 1997c, 366.

${ }^{8}$ GadAmER 1997b, 287. Véase también GW2, 228, 469; TM 269, 362-379, 491/GW1, 273, 368-384, 494.

$?$ KANT 1983, 53. 
tículo me gustaría hacer algunas observaciones sobre el potencial crítico y emancipador de la hermenéutica desde la perspectiva de los estudios literarios. Éste es un tema que, en mi opinión, todavía no ha recibido la atención que merece. Además, pienso que la significación de la hermenéutica filosófica para los estudios literarios no puede ser apreciada completamente cuando la discusión parte de una perspectiva metodológica demasiado simplista. Por otra parte, no estoy de acuerdo con la opinión de Joel Weinsheimer según la cual la hermenéutica de Gadamer, aun teniendo una orientación filosófica, "no tiene aplicaciones prácticas, ni consecuencias que puedan mejorar el ejercicio de la crítica literaria». ${ }^{10}$ Weinsheimer tiene por supuesto razón al señalar que la hermenéutica de Gadamer no proporciona ningún método para los estudios literarios, ni principios ni reglas de la interpretación válida. Sin embargo, me gustaría sugerir que la hermenéutica filosófica podría fomentar decisivamente la autocomprensión disciplinaria de los especialistas literarios. Pienso que no es insignificante para la práctica de la interpretación literaria și somos conscientes o no de la naturaleza y de las condiciones del proceso interpretativo del que nos ocupamos. Por lo tanto, pienso que los estudios literarios no deben desatender las cuestiones ontológicas, es decir, cuestiones referentes a la ontología de la existencia humana, del texto literario y de los estudios literarios. Por consiguiente, quisiera señalar algunos aspectos de la importancia para los estudios literarios de la reflexión hermenéutica sobre estas cuestiones ontológicas. Puesto que el espacio es limitado, me centraré en la dimensión crítica que la hermenéutica filosófica ofrece con sus descubrimientos acerca de la naturaleza histórica y dialógica de la existencia humana, así como su visión de las ciencias como prácticas de preguntar. ${ }^{11}$

10 WeINSHEIMER 1991, 24. Weinsheimer parece presuponer aquí que la aplicación se refiere necesariamente a cierta clase de aplicación mecánica de un sistema de reglas dado. Esta idea, sin embargo, contradice los hallazgos de Gadamer con respecto a la aplicación, a la que considera una tarea creativa que forma "parte integral del proceso hermenéutico" (TM 308/GW1, 313). Gadamer señala específicamente que la aplicación no "consiste en relacionar un universal ya dado con una situación particular", sino por el contrario en relacionar lo que uno ha entendido con la situación propia de cada uno (TM 324/GW1, 329).

${ }^{11}$ Una discusión más cuidadosa de la dimensión crítica de la hermenéutica debe aclarar el concepto de Gadamer de phrónesis y su tentativa de defender la razón práctica frente a la razón técnico-instrumental, para mencionar al menos un tema importante del que no me ocuparé aquí. Sobre este asunto véase por ejemplo WARNKE 2002a, 79-101; BERNSTEIN 2002, 273, 280; BERNSTEIN $1985,38-40,144-169,224-231$. 


\section{Sobre la universalidad de la comprensión}

La pretensión de universalidad gadameriana se basa en el "giro ontológico» que Heidegger impuso a la hermenéutica al considerar que la comprensión sigue la estructura de un círculo hermenéutico no ya como un principio metodológico sino como la manera humana de ser en el mundo. ${ }^{12}$ De tal modo, la interpretación - "comprender algo como algo" - se considera el fenómeno fundamental que caracteriza la estructura de toda la experiencia y el conocimiento humanos: nunca encontramos la realidad inmediatamente o "como tal", pues incluso las opiniones más simples implican el interpretar y el articular el mundo dentro de cierto horizonte de presuposiciones. ${ }^{13}$ Según la hermenéutica filosófica nos orientamos en el mundo desde un horizonte interpretativo constituido histórica, cultural, y socialmente que hace posible la experiencia y se transforma con nuevas experiencias, es decir, cuando encontramos en el mundo algo que no corresponde a nuestras presuposiciones. Además de la historicidad, Gadamer acentúa que "el ser-en-el-mundo del hombre es primordialmente lingüístico", y así "el lenguaje es el medio universal en el cual la comprensión tiene lugarm. ${ }^{14}$

La historicidad de la existencia humana significa para Gadamer que no podemos encontrar dentro de nosotros mismos la base de nuestro ser ni llegar a ser conscientes de todas nuestras presuposiciones: «Ser históricamente significa que el conocimiento de uno mismo nunca puede ser completom. ${ }^{15}$ Es decir, implica la finitud esencial de la existencia humana. Al mismo tiempo, sin embargo, la hermenéutica de Gadamer acentúa la posibilidad siempre abierta de ensanchar el horizonte de comprensión propio de cada uno y profundizar de tal modo en la autocomprensión de uno mismo. Esto tiene que ver con su opinión del lenguaje como algo que abre la posibilidad de diálogo y de com-

12 Véase Heidegger 1953 (1927), 148-153; TM 259-267/GW1, 264-271. Debe observarse que Gadamer (TM 264/GW1, 268) también pone de manifiesto la dimensión trascendental de este "giro ontológico»: "El problema de la hermenéutica se convierte en un objetivo universal, incluso logrando una nueva dimensión, gracias a la interpretación trascendental de la comprensión [de Heidegger]".

13 TM 90-92/GW1, 96-97.

14 TM 389, 443/GW1, 392, 447. Véase también GADAMER 1984, 58-60; GW2, 230, 237, 412; TM 474-476/GW1, 478-479.

is TM 302/GW1, 307. 
prensión mutua entre diversos universos de significado. ${ }^{16}$ Por otra parte, Gadamer concibe nuestro modo lingüístico de ser como esencialmente dialógico: así, "el lenguaje sólo alcanza su verdadero ser en diálogo". ${ }^{17}$ Esto no significa que el lenguaje sea un simple medio de comunicación sino más bien que es el medio en el cual el mundo se revela. Como lenguaje y mundo permanecen siempre juntos, Gadamer escribe sobre "lenguaje-mundos" y subraya que estos mundos nunca están cerrados: «En cuanto constituidos verbalmente, cada uno de estos mundos está siempre abierto a cualquier interpretación posible y por tanto a cualquier extensión de su propia imagen del mundo, y está por consiguiente disponible para otros». ${ }^{18}$ De hecho, piensa que nuestro modo lingüístico de ser implica que la visión del mundo de cada uno de nosotros está siempre en proceso de constituirse en diálogo con otros, en «la conversación que nosotros mismos somos". ${ }^{19}$

Estas premisas ontológicas básicas de la hermenéutica filosófica tienen importantes implicaciones para la filosofía de la ciencia. La pretensión de la universalidad de la hermenéutica se dirige en primer lugar contra las pretensiones positivistas y objetivistas de la universalidad de las ciencias naturales modernas que pretenden que la ciencia representa la realidad "objetivamente", "neutralmente", o "como tal", es decir, como una colección de hechos independientes del investigador. Esto supone una manifiesta ceguera ante el hecho de que las ciencias investigan inevitablemente la realidad desde perspectivas particulares, o como Gadamer apunta, a través de ciertas preguntas. Las preguntas abren una perspectiva que funciona como la condición de posibilidad para ver "hechos». ${ }^{20}$ Así, los hechos se constituyen en relación a un horizonte interpretativo particular o manera de orientarse a la realidad: existen solamente como respuestas a las pre-

${ }^{16} \mathrm{GW} 2,230$; TM 401-402/GW1, 405-406. Gadamer subraya que la razón no se desintegra debido a la multiplicidad de idiomas. Por el contrario, la lingüisticidad universal de la existencia humana exige la racionalidad universal que se manifiesta en el poder de la razón para elevarse "por encima de las limitaciones de cualquier lengua dada" (TM 402/GW1, 406).

17 TM 446/GW1, 449.

18 TM 447/GW1, 451. Según Gadamer el lenguaje implica «una orientación libre, y distanciada" respecto al mundo (TM 445/GW1, 448).

19 TM 378/GW1, 383. Posteriormente ha sido en especial Charles Taylor quien ha continuado trabajando en la idea hermenéutica de que la existencia humana es de naturaleza dialógica (véase TAYLOR 1991, 304-314).

${ }^{20} \mathrm{GW} 2,226,247,438-440$. 
guntas. Como Gadamer expone, lo que se piensa que existe en sí mismo es realmente "relativo a una manera particular de saber». ${ }^{21}$ Por consiguiente, "el Urphänomen hermenéutico" consiste en que "cada juicio tiene que ser considerado como la respuesta a una pregunta y que la única manera de comprender un juicio es encontrando la pregunta a la cual responde el juicio». ${ }^{22}$

Desde esta perspectiva no tiene ningún sentido oponer las ciencias humanas a las naturales en base a la oposición entre comprensión y explicación, porque ambas se basan en la interpretación de la realidad mediante preguntas. Por lo tanto, desde el punto de vista hermenéutico la investigación científica que hace uso de teorías y métodos debe ser considerada como la práctica de hacer preguntas a la realidad que se investiga. Estas preguntas desempeñan un papel esencial en la constitución del objeto de la investigación; así, no hay «objeto en sí mismo", sino solamente diversos aspectos del objeto que se revelan a la luz de diversas preguntas. ${ }^{23}$ Además, debido a nuestro modo histórico de ser, estas preguntas se limitan siempre a nuestro mundo histórico. Por lo tanto, la historicidad de la existencia humana implica que "comprendemos de diversa manera, si comprendemos algon. ${ }^{24}$

Según lo mencionado arriba, el fracaso del positivismo y el empirismo en la filosofía de la ciencia ha promovido la aceptación de la universalidad de la interpretación. Por ejemplo, Thomas Kuhn ha afirmado que también las ciencias naturales tienen una "base hermenéutica»: «En las ciencias naturales, igual que en las ciencias humanas, no hay ningún conjunto de categorías neutral, culturalmen-

21 TM 450/GW1, 454.

22 GADAMER 1981, 106; GADAMER 1977, 11/GW2, 226. Esta idea desempeńó un papel central ya en Verdad y método: "Comprender un significado es comprenderlo como la respuesta a una pregunta». (TM 375/GW1, 381).

${ }^{23}$ GW2, 238-239; TM 284-285, 301/GW1, 289, 306. Aunque se mantiene comúnmente que la hermenéutica filosófica rechaza el uso de teorías y de métodos, simplemente no es verdad. Por ejemplo, en su libro sobre Celan, Gadamer escribe: «Todos los métodos desarrollados por la academia pueden ser hermenéuticamente provechosos [...]" (GADAMER 1997d, 161). Y en otro lugar afirma que «sólo quien domine todos los métodos de su ciencia» tendrá éxito en el planteamiento de "preguntas reales, productivas" (GADAMER 1977, 12/GW2, 227). En vez de rechazar teorías y métodos, Gadamer cuestiona la autoconcepción metodológica de las ciencias modernas con el fin de hacer manifiestos los límites y las condiciones de posibilidad de las teorías y los métodos científicos.

${ }^{24}$ TM 297/GW1, 302. 
te independiente, dentro del cual la población — sea de objetos o de accionespueda ser descritas. ${ }^{25}$ Este giro también ha inducido a Habermas a que indique en su Teoría de la acción comunicativa: "Desde el punto de vista de la problemática del Verstehen, entonces, parece que las ciencias sociales no tienen en ningún caso un status especial». ${ }^{26}$ Pero incluso si Habermas acepta cierta universalidad de la estructura interpretativa en las ciencias, su opinión del conservadurismo de la hermenéutica permanece inalterada. Él no relaciona el poder emancipador de su teoría crítica con su base hermenéutica, sino que parece pensar que la reflexión emancipadora es posible a pesar de su base hermenéutica, es decir, debido a la distancia crítica que ofrecen los modelos de la explicación teórica. ${ }^{27}$

\section{La conciencia de ser afectado históricamente}

La universalidad de la hermenéutica implica para los estudios literarios que "la estética tiene que ser absorbida por la hermenéutica». ${ }^{28}$ Esto significa, en primer lugar, tomar en consideración la historicidad fundamental de toda comprensión, incluyendo la comprensión de la literatura, y de la experiencia del arte en general. El tratamiento de Gadamer de este tema constituye una dimensión central de su contribución a los estudios literarios - y a cualquier práctica académica-. Según Gadamer la comprensión tiene lugar siempre en una situación histórica limitada por un horizonte que está constituido por ciertas presuposiciones inconscientes, y este horizonte se amplía cada vez que uno llega a ser consciente de estas presuposiciones. Por lo tanto la condición más importante para esta extensión es que sabemos que tenemos siempre presuposiciones inconscientes. Gadamer piensa que esto requiere la superación de la oposición abstracta entre razón y tradición que hemos heredado de la Ilustración. Es peligroso cre-

25 KUHN 1991, 21.

26 Habermas 1984, 109. Además, siguiendo a Anthony Giddens, Habermas indica que las ciencias sociales tienen, en comparación con las ciencias naturales, "una tarea hermenéutica doble", pues la comprensión interpretativa en las ciencias sociales se relaciona no solamente con la dependencia de la teoría de la descripción de los datos sino también con la naturaleza del dominio del objeto como realidad social ya simbólicamente estructurada. Esto se aplica naturalmente a todas las ciencias humanas. Véase HABERMAS 1984, 109-110; GIDDENS 1976, 146, 158; GIDDENS 1984, 221, 225-230; BOHMAN et al. 1991, 5.

27 Cf. Habermas 1986a, 266-267; Habermas 1971, 129-130; Habermas 1984, 1-7; MCCARTHY 1984, 191-3.

28 TM 164/GW $1,170$. 
er en la razón o la libertad absolutas, es decir, en la posibilidad de liberarse de todas las tradiciones y presuposiciones, pues precisamente eso da lugar a nuestra ceguera respecto a ellas. Gadamer también cuestiona la idea de que pertenecer a una tradición implicaría una restricción de la libertad: « No está toda existencia humana, incluso la más libre, limitada y calificada de varias maneras? „2 ${ }^{29}$ Para él, pertenecer a una tradición "no es tanto una condición que limite sino la que hace posible la comprensión». ${ }^{30}$ En la existencia humana la razón y la tradición están siempre entrelazadas y mutuamente condicionadas: la razón nunca funciona en un vacío sino en la historia, respecto a ciertas circunstancias históricas, y la tradición nunca es la transmisión ciega del pasado a las generaciones futuras: como Gadamer expone, siempre hay «elementos de la libertad» en la tradición. ${ }^{31}$

Por lo tanto, Gadamer desea sustituir el pensamiento dicotómico ilustrado por la conciencia hermenéutica de la historicidad de la comprensión. Esto significa tener conciencia del hecho de que encontramos al mundo, a la gente y a nosotros mismos siempre dentro de un horizonte interpretativo dado que no podemos controlar totalmente: venimos a un mundo siempre ya interpretado, y nunca podemos darnos cuenta de todas las premisas de nuestro pensamiento y modo de actuar. Para Gadamer "la conciencia de ser afectado por la historia" (wirkungsgeschichtliches Bewusstsein) forma la base de la reflexión emancipadora. Piensa que sólo cuando uno se da cuenta de que la realidad nunca es aprehendida inmediatamente, sino siempre a través de conceptos, preguntas y perspectivas cultural e históricamente formados, estos conceptos, preguntas y perspectivas pueden ser reflejados, discutidos, y superados. De otro modo uno se aferra a ellos ciega y dogmáticamente, y sigue siendo así su prisionero. ${ }^{32}$ Del mismo modo, la hermenéutica tiene un significado emancipador como filosofía de la ciencia que muestra sobre qué clase de preguntas, presuposiciones y variados intereses prácticos se orientan las ciencias hacia la realidad y producen conocimiento. Como Gadamer apunta, tal reflexión hermenéutica puede, «al hacer transparentes las precom-

$\begin{array}{ll}{ }_{29} & \text { TM 276/GW1, } 280 . \\ 30 & \text { TM 329/GW1, } 334 . \\ { }_{31} & \text { TM 281/GW1, } 286 . \\ 32 & \text { Es interesante que }\end{array}$

${ }^{32}$ Es interesante que también HABERMAS (1984, 48-51, 70-72) considere que la conciencia de que la realidad es vista siempre desde cierta perspectiva es una condición para el discurso racional, pues sólo entonces se pueden comparar las varias perspectivas, evaluarlas y discutirlas críticamente. 
prensiones dominantes de las ciencias, abrir nuevas dimensiones al preguntar y servir de tal modo indirectamente al trabajo metodológicom. ${ }^{33}$

Desde la perspectiva hermenéutica el sentido de la historicidad fundamental de nuestro ser es una fuente de reflexión emancipadora y crítica porque lo que se produce históricamente es producido por la acción humana, y por lo tanto podría también haber sido de otra manera y está sujeto a crítica. Así, el potencial crítico de la hermenéutica se apoya esencialmente en su reflexión en la historicidad de todas las clases de órdenes, instituciones y prácticas humanas. Como Manfred Frank señala, la idea fundamental de la hermenéutica es "que los órdenes simbólicos, al contrario que las leyes naturales, se fundan en interpretaciones; por lo tanto sólo pueden reclamar una existencia hipotética, pueden ser transformadas y transgredidas por nuevas proyecciones de significador. ${ }^{34} \mathrm{La}$ hermenéutica nos recuerda que los sistemas culturales y sociales que nos rodean no tienen ningún fundamento necesario $o$ "natural», y que se pueden por tanto transformar en sistemas más justos mediante la evaluación y la discusión críticas. Comprender qué clases de procesos y de tradiciones históricos han producido el mundo contemporáneo facilita a su vez tal evaluación crítica.

Debe observarse que, a pesar de sus muchas diferencias, Gadamer y Habermas confian en el pensamiento crítico que cuestiona maneras represivas aparentemente evidentes en sí $o$ aparentemente naturales del pensar y el actuar revelando su naturaleza histórica y contingente. ${ }^{35}$ Además, este enfoque de la tarea crítica de la filosofía contemporánea se aproxima a la idea de Michel Foucault de la filosofía crítica en la cual «la crítica de lo que somos es al mismo tiempo el análisis histórico de los límites que se nos han impuesto y un experimento acerca de la posibilidad de ir más allá de ellosm. ${ }^{36}$ Como es bien sabido, en sus últimos escritos, Foucault declaró que él continúa el proyecto emancipador de la filosofía de la Ilustración analizando la "ontología histórica de nosotros mismos», es decir, esos procesos y condiciones históricos que nos constituyen en sujetos. ${ }^{37}$ Sin embargo, pienso que la hermenéutica filosófica aclara más a fondo que la mayoría del resto de las formas de filosofía contemporánea crítica cómo podemos desarrollar

\footnotetext{
${ }^{33}$ GW2, 248. Véase también GADAMER 1977, 10-11/GW2, 225-226; TM 301/GW1, 306.

34 FRANK 1989, 6.

Cf. Habermas 1986b, 198; Gadamer 1981, 149-150; GW2, 467-469.

36 Foucault 1984, 50.

37 Foucault $1984,45,49$.
} 
tal conocimiento de nuestra propia historicidad, y superar de tal manera las prácticas que nos resultan problemáticas. Mientras que Foucault esquiva la pregunta referente a las condiciones de posibilidad de su propia investigación, y Habermas parece confiar en la razón ahistórica ilustrada, Gadamer, por el contrario, logra ilustrar las condiciones de posibilidad para la conciencia reflexiva emancipadora de la propia historicidad sin tener que recurrir a la razón ahistórica. ${ }^{38}$

Para Gadamer la reflexión crítica y emancipadora, como toda comprensión, tiene lugar dentro de una situación histórica. Además, no puede ser absoluta: no puede preguntar todo, ni conducir al perfeccionamiento de la autotrasparencia de un individuo o de una sociedad. Desde la perspectiva hermenéutica la emancipación no significa la emancipación perfecta de la tradición sino tomar conciencia de la propia situación histórica. Sin embargo, como Gadamer precisa, es difícil adquirir conciencia de que «la mera idea de una situación significa que no estamos fuera de ella y que por lo tanto no podemos tener ningún conocimiento objetivo de ella", lo cual hace que entender esta situación sea "una tarea que nunca se acaba del todo". ${ }^{39}$ La situación está limitada por un horizonte, pero este horizonte no está constituido por un conjunto estable de presuposiciones dadas. Por el contrario, está en proceso de ser constantemente remodelado, pues constantemente tenemos que probar nuestras presuposiciones. Según Gadamer, encontrar mundos de sentido ajenos, por ejemplo obras de arte, desempeña un papel decisivo en esta prueba. Opina que tales mundos pueden funcionar como "provocación", haciendo visibles las presuposiciones que nos afectan normalmente de manera inconsciente. ${ }^{40}$ Para él, es precisamente el encuentro del otro el que ofrece la posibilidad de descubrir cosas que nos han determinado «a nuestras espaldas», y valorar críticamente "otras posibilidades», justificadas e injustificadas en la propia precomprensión. ${ }^{41}$

\section{El significado emancipador de encontrar al otro}

Realmente, Gadamer y Habermas no difieren demasiado tanto en la cuestión referente al significado y la posibilidad de la reflexión emancipadora como en sus

38 WARNKE 2002b, 318-320.

39 TM 301/GW1, 307.

40 TM 299/GW1, 304.

41 Cf. GW2, 244, 247; GADAMER 1997a, 45; TM 298-299/GW1, 304; RJCOEUR 1991, 283; BERNSTEIN 1985, 151-154. 
opiniones sobre su naturaleza y condiciones de posibilidad. Habermas insiste en la necesidad de la explicación "monológica" con los modelos teóricos, que permiten superar la comprensión dependiente del contexto; por consiguiente, sostiene que la hermenéutica "dialógica», "no es capaz de apreciar el poder de la reflexión». ${ }^{42}$ Gadamer, por su parte, no entiende la reflexión emancipadora como un acto autosuficiente de la razón sino más bien como algo que se vuelve posible solamente gracias al encuentro dialógico del otro: «La autocomprensión siempre se produce a través de la comprensión de algo distinto del sí mismo». ${ }^{43}$ Este otro puede ser cualquier persona o cualquier cosa que abra un mundo de significado ajeno.

El significado emancipador del encuentro hermenéutico de universos de significado histórica o culturalmente distantes se basa en el hecho de que estar familiarizado con modos ajenos de pensar y de actuar nos permite tomar distancia crítica respecto a las prácticas dominantes y en apariencia naturales del propio mundo histórico. ${ }^{44}$ Tales encuentros hacen perceptible el horizonte interpretativo desde el que cada cual se orienta al mundo y los prejuicios que uno da por sentado. Así, como Thomas McCarthy señala, la autorreflexión crítica es para Gadamer «algo que no se opone a la comprensión», como parece ser para Habermas, sino más bien "un momento integrante» del intento de comprender el punto de vista del otro. ${ }^{45}$ De hecho, Gadamer postula que "comprendemos al máximo" precisamente cuando un diálogo exitoso nos permite ver más allá de nuestras presuposiciones anteriores y superarlas. ${ }^{46}$ En tal diálogo, el profundizar en la propia autocomprensión está unido a entender otras visiones del mundo y formas de vida. Es un proceso que ensancha el sentido de la propia conciencia acerca de otras posibilidades de ser y agranda así el espacio de libertad en el cual uno puede tomar decisiones fundamentales referentes a la propia Weltanschauung y la manera de ser en el mundo.

Muchos comentaristas han visto el papel fundamental del otro en la hermenéutica de Gadamer como lo que la distingue decisivamente no sólo de Habermas sino también de Heidegger. ${ }^{47}$ En Sein und Zeit, de Heidegger, el ser con otros

\footnotetext{
42 Habermas 1986a, 268; Habermas 1971, 129-134.

43 TM 97/GW1, 102.

${ }^{44}$ Cf. TAYLOR 2002b, 295-296.

45 MCCARTHY 1984, 189.

46 GW2, 243.

47 Véase por ejemplo DOSTAL 2002, 255-257.
} 
(Mitsein) significa sobre todo ser inauténtico (uneigentlich), mientras que Gadamer piensa que lo que aprendemos en diálogo con otros no sólo es la comprensión de nuestra propia historicidad, sino también la superación de nuestros límites, con el fin de poder encontrar ahí las máximas posibilidades propias de nuestro ser. ${ }^{48}$ Además, la concepción de Gadamer de la reflexión emancipadora, que enfatiza el significado de encontrar un mundo de sentido extranjero, se puede considerar como particularmente relevante en nuestro mundo multicultural contemporáneo. ${ }^{49}$ Esto es valido también para su visión de la naturaleza del diálogo auténtico: lo caracteriza como un movimiento que procede mediante preguntas y respuestas, en el que los que dialogan no procuran defender solamente su propia posición sino luchar para la comprensión mutua que sobrepasa las opiniones iniciales de ambos. Gadamer defiende un ideal del diálogo crítico basado en el arte de preguntar y de estar abierto a las preguntas, y sugiere que tal diálogo permite "una actitud crítica hacia todas las convenciones". ${ }^{50}$ No acepta una idea relativista de la inconmensurabilidad de las diversas visiones del mundo y formas de vida —o la visión de que somos siempre prisioneros de nuestras convencionessino que piensa que la lingüisticidad universal del ser-en-el-mundo humano permite siempre el diálogo crítico. ${ }^{51}$ Creo que la conciencia hermenéutica del modo dialógico del ser humano - la conciencia de la manera en que nos convertimos en nosotros mismos solamente en relación con otros- aumenta la posibilidad de diálogo exitoso al ayudarnos a ver que tenemos que escuchar al otro si deseamos comprender en general.

Pero ¿qué clase de otro es el texto literario que se encuentra en los estudios literarios? Gadamer es famoso por su crítica de la conciencia estética. En esta crítica es crucial el rechazo de "su neutralización de la cuestión de la verdad", es decir, la renuncia a la idea de que el arte sea un objeto de experiencia estética definido en términos subjetivos, como algo que proporciona un placer sensual momentáneo. ${ }^{52}$ Gadamer escribe contra la conciencia estética de que «el arte sea conocimiento y experimentar una representación artística signifique compartir

48 Véase GW10, 97-98; HEIDEGGER 1953 (1927), 125-130.

49 A este respecto, pienso que Taylor tiene razón al evaluar la contribución de la filosofía de Gadamer desde el siguiente punto de vista: «El gran desafío de este siglo, tanto para la política como para las ciencias sociales, es comprender al otro" (TAYLOR 2002b, 279).

50 GW2, 204.

${ }^{51}$ GADAMER 1997a, 29, 32; GW2, 230. Véase también TAYLOR 2002a, 142.

52 TM 99/GW1, 105. 
dicho conocimiento». ${ }^{53}$ Opina que la experiencia de la verdad inducida por una representación artística no se puede formular en términos de "conocimiento definitivom: consiste más bien en la interpretación de algo, en la comprensión de una nueva manera de pensamiento con sentido, y, si es una experiencia genuina, «no dejará sin cambios a quien lo haya experimentadom. ${ }^{54}$ Contra la diferenciación estética que abstrae una representación artística de su contexto histórico, Gadamer acentúa que las representaciones artísticas pertenecen al mundo: «Encontramos una obra de arte en nuestro mundo histórico, y encontramos en ella un mundo de la representación artística a través de la cual aprendemos a comprendernos a nosotros mismoss. ${ }^{55}$ De este modo, cada encuentro genuino del texto literario en su alteridad implica tanto un autoencuentro como una autoalteración. ${ }^{56}$

Así, desde la perspectiva de la hermenéutica gadameriana, encontrar el texto en calidad de otro es una tarea central de los estudios literarios. Esta tarea, sin embargo, supone un auténtico desafío. Según lo sugerido arriba, encontrar al otro da lugar al conocimiento de la historicidad y de la parcialidad fundamentales de la propia comprensión pero, por otra parte, sólo quién tiene tal conocimiento puede ser sensible a la alteridad del otro: «lo importante es conocer el sesgo propio, de modo que el texto pueda presentarse en toda su alteridad y afirmar así su propia verdad contra los significados previos y los prejuicios de cada uno». ${ }^{57}$ Es así cuando se plantea la pregunta: ¿cómo se consigue acceder a tal círculo virtuoso? Según Gadamer, se requiere la apertura "fundamental de mantenerse abierto a la conversación", que él considera el "principio supremo" de la hermenéutica. ${ }^{58}$ Gadamer sugiere que uno sólo puede hacer justicia al texto en calidad de otro cuando se aproxima al mismo con dicha apertura, «de tal manera que tenga algo que decirme»; es decir, "como un genuino compañero de diálogon."

\footnotetext{
${ }^{53}$ TM 97/GW1, 103.

54 TM 100/GW1, 105-106.

55 TM 97/GW1, 102. la aprehensión del otrom. (JAUSS 1989, 208-209).

57 TM 269/GW1, 273-274. Cf. WARNKE 2002a, 94-99.

58 GADAMER 1997a, 36.

59 TM 358, 361/GW1, 364, 367.
}

${ }^{56}$ Hans Robert Jauss expresa este hallazgo central de la hermenéutica literaria escribiendo que el texto literario "preserva y revela la historicidad del otro" de tal modo que hace posible que otras maneras de experimentar el mundo usean comprensibles como la aprehensión de uno mismo en 


\section{El arte del preguntar crítico}

Algunos especialistas han entendido la concepción dialógica de Gadamer acerca de la interpretación del texto como una demanda para aceptar acríticamente lo que dice el texto. Tal posición, sin embargo, deriva de un grave malentendido. Desde la perspectiva gadameriana los estudios literarios aparecen, por el contrario, como la práctica del arte del preguntar crítico. Como mencioné arriba, la visión hermenéutica de las ciencias pone de manifiesto su naturaleza como prácticas de plantear preguntas. Sin embargo, son generalmente inconscientes de constituir tales prácticas. Gadamer menciona como ejemplo las estadísticas, que «son unos excelentes medios de propaganda porque dejan hablar a los 'hechos' y por lo tanto simulan una objetividad que en realidad depende de la legitimidad de las cuestiones preguntadasm. ${ }^{60} \mathrm{La}$ hermenéutica filosófica pretende mejorar la autocomprensión de las ciencias de modo que puedan convertirse en prácticas autoconscientes de preguntar. Gadamer se centra en la exposición de cómo las ciencias humanas siguen "la lógica de la pregunta». ${ }^{61}$

Según la hermenéutica filosófica los estudios literarios se muestran como una práctica dialógica que consiste en preguntar y responder. El proceso interpretativo comienza cuando el texto plantea una pregunta al intérprete. Como vimos arriba, oír esta pregunta inicial requiere una apertura fundamental al texto en calidad de otro. Una vez interpelado por esta pregunta, el intérprete, a su vez, responde comenzando a plantear preguntas al texto, y estas preguntas provienen inevitablemente de su propio mundo histórico. Por tanto, la distancia y la tensión entre el texto y el intérprete pertenecen íntegramente al proceso de preguntar:

La tarea hermenéutica consiste en no encubrir esta tensión procurando una asimilación naïve de los dos sino en revelarla conscientemente. Esta es la razón por la cual es parte del enfoque hermenéutico proyectar un horizonte histórico que sea diferente del horizonte presente. La conciencia histórica conoce su propia alteridad y por lo tanto enfoca el horizonte del pasado desde el suyo propio. $^{62}$

\footnotetext{
60 TM 301/GW1, 306.

${ }^{61} \mathrm{TM} 370 / \mathrm{GW} 1,375$.

${ }^{62}$ TM 306/GW1, 311.
} 
Es decir, la distancia al texto no se debe entender como un obstáculo a la comprensión sino, por el contrario, como algo que permite entender el texto de forma diferente, y en un contexto más amplio que el de sus contemporáneos, sacando de este modo nueva luz de él. Así pues, no es de ninguna manera la tarea del intérprete la comprensión del texto como su autor o sus contemporáneos lo entendieron: tal y como Gadamer afirma, mantener un diálogo con el texto implica siempre "pensar sobre lo que el autor aceptaba incuestionablemente y por lo tanto no consideraba». ${ }^{63}$ Aunque Gadamer ha sido siempre absolutamente claro sobre este punto, parece repetirse el obstinado malentendido de que el acercamiento hermenéutico está inevitablemente limitado de alguna manera a comprender la autocomprensión del objeto de investigación. ${ }^{64}$ Sin embargo, desde la perspectiva hermenéutica el objeto de investigación de las ciencias humanas, bien sea un texto literario o una forma de vida cultural o históricamente distante, está constituida por una realidad simbólica que conlleva ciertas maneras de interpretar y de formar el mundo, y está claro que el objeto de investigación puede tener solamente un conocimiento bastante limitado de sus maneras de revelar el mundo o de pertenecer a cierto mundo histórico. Como Madison ha remarcado, las ciencias humanas hermenéuticamente orientadas practican la interpretación de las autointerpretaciones de agentes, textos y formas culturales de vida, y tal interpretación requiere la explicación no «de las intenciones sino de la lógica de ese orden en cuestión (texto, cultura, etc)m. ${ }^{65}$

Puesto que con frecuencia se ha descuidado el elemento crítico que define el proceso hermenéutico de la interpretación, Gadamer ha tratado específicamente este punto en sus últimos escritos:

Asumir que el énfasis en el factor esencial de la tradición que interviene en toda comprensión implica una aceptación acrítica de la tradición y un conservadurismo sociopolítico constituye un grave malentendido [...]. En verdad la confrontación de nuestra tradición histórica es siempre un desafio crítico de esta tradición. ${ }^{66}$

${ }^{63}$ TM 374/GW1, 380.

${ }^{64}$ Por ejemplo, tal opinión la expresa, algo asombrosamente, WARNKE $(1987,116)$, que pretende que la hermenéutica gadameriana "permanece atada a la autocomprensión, explícita o implícita, de cada sociedad".

${ }^{65}$ MADISON 1997, 351, 359.

${ }^{66}$ Gadamer 1987, 87. 
Sin embargo, este proceso crítico de preguntar no se dirige solamente al mundo histórico del texto: a saber, también se dirige al mundo histórico del intérprete. Gadamer acentúa que en el proceso de comprender el investigador no solamente pone en duda el texto sino que también se expone a sí mismo al texto hasta el punto de llegar a ser desafiado por él. Así, en los estudios literarios que se orienten desde una perspectiva hermenéutica, el texto no se toma, sin más, en serio, sino que también se examina críticamente. Es decir, el preguntar crítico que forma su esencia es doble: por un lado, se dirige a las presuposiciones y las asunciones no dichas que permanecen detrás del texto; por otra parte, se dirige al propio mundo histórico de cada uno.

Gadamer piensa que el horizonte del texto literario es esencialmente el horizonte de la pregunta a la que el texto se puede considerar como respuesta. El intérprete debe esforzarse en reconstruir este horizonte que presta al texto su significado. Sin embargo, Gadamer precisa que el horizonte de esta pregunta no existe en sí mismo sino solamente en relación al horizonte histórico del intérprete. Se fusiona con el proceso de preguntar en el que el intérprete está envuelto. ${ }^{67}$ Los planteamientos de Gadamer sobre la naturaleza de la pregunta hacen más inteligibles esta compleja dialéctica de preguntas y respuestas. Indica que "la verdadera y fundamental naturaleza de una pregunta" consiste en "poner de manifiesto las posibilidades indeterminadas de una cosam. ${ }^{68}$ Esto nos recuerda que el texto versa siempre sobre algo: puede hablar sobre, por ejemplo, amor o desesperación, y proporcionar a gentes del pasado o de otra cultura ciertas posibilidades de pensar sobre tales temas. Pero el intérprete no puede limitarse a intentar imaginar lo que significó el texto para su auditorio inicial. Debe descubrir qué podría significar el texto para la gente de su propio mundo. Gadamer considera este "problema de la aplicación" como parte integral de la tarea de la interpretación: el intérprete tiene que reflejar qué posibilidades de ser abre el texto para sus contemporáneos.

Cuando la investigación literaria sigue esta dialéctica del preguntar resulta claro que la tarea de interpretar las autointerpretaciones no puede significar objetivarlas, dar una explicación neutral del texto desde la distancia. Por el contrario, el intérprete debe dejar al texto relacionarse consigo, poniendo en duda de

\footnotetext{
${ }^{67}$ TM 374/GW1, 379-380.

${ }^{68}$ TM 375/GW1, 380.
} 
ese modo las cosas que da por sentadas. El texto funciona como una pregunta cuando hace que algunas opiniones que el intérprete ha llevado a cabo aparezcan como cuestionables, y de tal modo le ayuda a descubrir nuevas posibilidades de pensamiento. ${ }^{69}$ Por otra parte, las preguntas que el intérprete plantea al texto pueden hacer que algo que el texto muestra como autoevidente aparezca como cuestionable. Lo que articula todo este proceso de preguntar es el hecho de que abre nuevas perspectivas, es decir, nuevas posibilidades de dar significado a las cosas: "Preguntar abre posibilidades de sentido». ${ }^{70}$ Los estudios literarios, por tanto, y las ciencias humanas en gran medida, se pueden considerar como prácticas críticas que promueven el Fragwürdige, lo cuestionable y lo que vale la pena preguntar, al margen de lo que parezca ser autoevidente, en el pasado y en el presente. ${ }^{71}$

\section{Los estudios literarios como reveladores de nuevas posibilidades de ser}

Para aclarar en mayor medida el papel que se abre para los estudios literarios a la luz de la discusión precedente, quisieran volver una vez más a la ontología del texto literario. Según Gadamer una representación es una estructura significativa que se debe interpretar siempre de forma diferente, y ésta adquiere su "propio ser" solamente en estas interpretaciones mediadas: la temporalidad de las representaciones artísticas significa que "existe solamente siendo siempre algo diferente». ${ }^{72}$ En la interpretación, las posibilidades de ser de la representación artística "emergen, mientras la obra se explica a sí misma, tal y como estas eran, en la variedad de sus aspectos". ${ }^{73}$ Así, la interpretación no es algo externo al texto literario, sino que pertenece a su mismo ser. Gadamer se refiere a esto como la "no-diferenciación de la mediación (Vermittlung) de la obra en sí misma». ${ }^{74}$

69 Sobre la idea del texto como pregunta, cf. Figal $2000,337$.

70 TM 375/GW1, 381.

71 Cf. GADAMER 1977, 12/GW2, 227. Aquí Gadamer afirma que no solamente la apertura fundamental sino también la imaginación son decisivas para «el sentido de lo que es cuestionable [Fragwürdige]" y «la capacidad de plantear preguntas reales, productivas, algo en lo cual, hablando en general, solamente quién domina todos los métodos de su ciencia tiene éxito".

72 TM 117, 123/GW1, 123, 128.

73 TM 118/GW1, 123. En otra parte Gadamer caracteriza la comprensión como un acontecimiento en el cual el significado "toma forma y se actualiza" (TM 165/GW1, 170).

${ }^{74}$ TM 120/GW1, 125. 
Gadamer entiende la obra literaria como «un acontecimiento del ser» (Seinsvorgang) en el cual ciertas posibilidades del ser vienen al lenguaje: esto es el «volverse-presente del sern. ${ }^{75}$ Además, sugiere que todos los textos escritos, es decir, también los textos académicos, comparten en el modo de ser de la literatura. ${ }^{76}$ Sin embargo, parece haber una relación particularmente cercana entre los modos de ser de los textos literarios y los textos académicos sobre literatura, dado que los textos literarios existen correctamente solamente gracias a sus interpretaciones. Desde esta perspectiva los estudios literarios se pueden considerar como prácticas de explicar, articular y analizar textos literarios para abrir nuevas posibilidades de ser para la gente de nuestro mundo contemporáneo.

Esta perspectiva que la hermenéutica de Gadamer proporciona sobre la tarea crítica y emancipadora de los estudios literarios se ha utilizado y se ha desarrollado aún más allá en los estudios de su seguidor francés más importante, Paul Ricoeur. Este reputa como tarea primordial de los estudios literarios la de articular las posibilidades de ser abiertas por los mundos que los textos literarios proyectan: "Comprender un texto [...] no es encontrar un sentido sin vida que este contenga, sino revelar la posibilidad de ser indicada por el texton." Además, Ricoeur ha relacionado de manera productiva este proceso de la interpretación literaria con la problemática de la construcción de la identidad. Explica cómo con la lectura de textos literarios reinterpretamos y reconstituimos constantemente nuestra identidad en relación a las posibilidades reveladas por los textos: de tal modo, nuestras opiniones sobre cómo podemos vivir nuestras vidas, quiénes somos, y quiénes queremos ser, se amplían y se enriquecen. Cuando el lector se expone a sí mismo al mundo propuesto por el texto, recibe "de este un sí mismo aumentado», o, para utilizar otra expresión ricoeuriana, encuentra "variaciones imaginarias» del sí mismo. ${ }^{78}$

75 TM 144, 159, 378/GW1, 148-149, 165, 384.

76 TM 162-163/GW1, 167-168.

77 RICOEUR 1991, 66. Las posibilidades de ser desempeñan un papel tan central en la hermenéutica de Ricoeur que Madison escribe: «En su hermenéutica del sujeto Paul Ricoeur ha logrado dibujar una verdadera poétique du possiblen (MADISON 1995, 89).

${ }^{78}$ RICOEUR 1991, 88. Ricoeur escribe sobre el encuentro del mundo del lector y el mundo del texto, pero no sugiere, como Gadamer, que el lector en este proceso ponga en juego sus propias presuposiciones, sino que por el contrario el lector debe «irrealizarse" o "potencializarse" a sí mismo: "Como lector, me encuentro a mí mismo solamente perdiéndome» (RICOEUR 1991, 88). Esta idea de perderse parece problemática desde un punto de vista gadameriano, que subraya nues- 
Esta es una perspectiva que Gadamer retoma siguiendo la idea aristotélica de que el arte apunta a «la dimensión de lo posible, y por lo tanto también a la crítica de la realidad» ${ }^{79}$ Ricoeur ha reformulado esta idea como sigue: «El poder del texto de abrir una dimensión de la realidad implica en principio un recurso contra cualquier realidad dada y de tal modo la posibilidad de una crítica de lo real. En el discurso poético es donde este poder subversivo está más vivo» ${ }^{80}$ Como Ricoeur hace notar, este tema crítico está ya presente en la noción heideggeriana del comprender como "la proyección de mis propias posibilidades; esto significa que el modo de ser del mundo abierto por el texto es el modo de lo posible, o mejor, del poder-ser: en esto reside la fuerza subversiva de lo imaginariom. ${ }^{81}$ El tratamiento de Ricoeur de esta fuerza subversiva de lo imaginario constituye una dimensión central y uno de los aspectos más originales de su hermenéutica. Por ejemplo, explica con gran surileza el papel crucial que desempeñan los textos literarios en el desarrollo de nuestra imaginación ética, que a su vez constituye una dimensión central de nuestro ser-en-el-mundo. ${ }^{82}$ Los textos literarios pueden dar forma a nuestras opiniones sobre qué es lo que constituye una buena vida y funcionan como "provocación para ser y actuar de diferente maneraw. ${ }^{83}$

Gadamer y Ricoeur relacionan el significado crítico y emancipador de comprenderse a uno mismo por medio de la literatura con la idea de la distancia. Gadamer afirma que la representación artística proporciona «la distancia necesaria para ver", distancia que nos permite ver "la verdad de nuestro propio mundo", en el que "nos reconocemos a nosotros mismos». ${ }^{84} \mathrm{De}$ igual manera, Ricoeur escribe: "el distanciamiento, en todas sus formas y figuras, constituye el momento crítico

tra incapacidad para introducirnos en un mundo ajeno y nos recuerda siempre la mediación entre nuestro mundo histórico y el mundo del texto.

79 TM 579/GW2, 478.

${ }^{80}$ RiCOEUR 1991, 300.

81 RICOEUR 1991, 300.

${ }^{82}$ Ricoeur 1994, 164ss. Véase también KEMP 1995, 371-394. En la filosofía de Ricoeur el problema de la imaginación está estrechamente relacionado con el problema de la creatividad, que él pretende que es el problema más importante de la totalidad de su filosofía (RICOEUR 1981, 165). Tal y como señala Jervolino, este problema apunta, dentro de la filosofía de Ricoeur, a la condición humana, a "los seres humanos, que actúan y sufren, y en su actuar y su sufrimiento, en su interacción y compasión, están llamados a inventar, que es encontrar y producir (la palabra 'inventar' contiene este doble sentido) el sentido de sus vidas" (JERVOLINO 1995, 535).

${ }^{83}$ RICOEUR 1990, 249.

${ }^{84}$ TM 128/GW1, 133. 
de la comprensión por excelencia». ${ }^{85}$ Ambos piensan que, cuando encontramos un mundo proyectado por un texto literario, nos imaginamos cómo sería la vida en él, y mediante este proceso tomamos distancia respecto a nosotros mismos y a las prácticas que damos por sentadas. Al principio su Verdad y método, Gadamer analiza esta idea de la distancia crítica en lo referente a la idea de Bildung, que le parece que merece de nuevo atención. Sostiene que la Bildung, con la cual Herder se refería a un proceso continuo de autoformación y cultivo a través de la cultura, se caracteriza tanto por una capacidad de tomar distancia con respecto a lo más próximo a uno mismo, como por cierta receptividad y apertura a perspectivas diversas. Es decir, en la Bildung la capacidad de «distanciarse uno de sí mismo» va uni$\mathrm{da}$ a la conciencia de los "puntos de vista de posibles otros». ${ }^{86}$ Quisiera sugerir que los estudios literarios, que revelan diversas posibilidades de ser, son una manera significativa de interpretar y continuar este ideal.

En conclusión, la dimensión de lo posible desempeña un papel central en el pensamiento hermenéutico, y define también la concepción hermenéutica de la libertad; algo que Günter Figal ha aclarado con perspicacia:

Ser libres consiste en que en el mundo nos abrimos a las posibilidades que nos resultan accesibles. Estamos abiertos a las posibilidades abiertas del mundo. Esta apertura, que Heidegger en Ser y tiempo designó con el concepto de "revelación" [Erschlossenheit], es nuestra manera de ser. ${ }^{87}$

Según esta concepción de la libertad, la capacidad de aprehender diversas posibilidades de pensar y de ser resulta de suma importancia emancipadora. Del mismo modo, esforzarse por una mayor libertad aparece como parte integral del proceso de la comprensión hermenéutica. Esa copertenencia mutua estaba ya implicada en la noción heideggeriana de la comprensión, noción que Gadamer recoge y elabora con más detalle: "Así es verdad en cada caso que una persona que comprende, se comprende a sí misma (sich versteht), proyectándose sobre sus posibilidades» ${ }^{88} \mathrm{La}$ dimensión emancipadora de la hermenéutica debe enten-

\footnotetext{
85 RICOEUR 1991, 37.

${ }^{86}$ TM 17/GW1, 22-23.

${ }^{87}$ FIGAL 1998, 18.

88 TM 260/GW1, 265.
} 
derse en relación a esta tesis hermenéutica central: que toda comprensión implica la autocomprensión; como Gadamer afirma, la tarea de la hermenéutica es "hacer que las personas se autocomprendan» de tal manera que "ello nos ayude a ganar nuestra libertad respecto a todo lo que se ha convertido en incuestionablew. ${ }^{89}$ Así, aunque Gadamer no cree en la idea abstracta de la libertad autosuficiente que depende de la utopía de despojarse a uno mismo de toda historicidad, la noción de la libertad que se basa en la autocomprensión, la aprehensión de diversas posibilidades de ser, y la interpretación de la propia historicidad forman el corazón de su hermenéutica.

Para concluir, espero haber demostrado que la hermenéutica gadameriana proporciona gran número de planteamientos críticos y emancipadores que valdría la pena desarrollar mejor. Quizás en última instancia lo que subyace a los planteamientos aquí discutidos es cierta clase de "êthos hermenéutico" caracterizado por la voluntad de comprender al otro, el perseguir la sinceridad intelectual y el estar preparado para reconocer la propia finitud y permanente incompletud. Así, de alguna manera el potencial crítico de la hermenéutica brota en última instancia de la sabiduría socrática de saber que uno no sabe. Para Gadamer esta docta ignorantia apunta a la visión de que solamente quién entiende su finitud fundamental puede plantear preguntas auténticas - es decir, preguntas que abren nuevas posibilidades de ser y de pensar-. Y $Y$ desde la perspectiva hermenéutica toda investigación crítica significativa sobre la existencia humana, tanto a través de textos literarios como de cualquier otra forma, puede caracterizarse como el arte de preguntar: «El arte de preguntar es el arte de preguntar siempre más, es decir, el arte de pensar». ${ }^{90}$

\section{Bibliografía}

R. BERNSTEIN, Beyond Objectivism and Relativism: Science, Hermeneutics and Praxis. Filadelfia: University of Pennsylvania Press, 1985.

R. BERNSTEIN, «The Constellation of Hermeneutics, Critical Theory, and Deconstruction». - Robert Dostal (ed.), The Cambridge Companion to Gadamer. Cambridge: Cambridge UP, pp. 267-282, 2002.

\footnotetext{
89 GADAMER 1986, 149-150.

${ }^{90}$ TM 367/GW1, 372.
} 
J. BOHMAN, D. Hiley \& R. Shusterman, «Introduction: The Interpretive Turn». David Hiley, James Bohman \& Richard Shusterman (eds.), The Interpretive Turn. Philosophy, Science, Culture. Ithaca: Cornell UP, pp. 1-14, 1991.

R. BUBNER, Dialektik und Wissenschaft. Fráncfort del Meno: Suhrkamp, 1973.

R. DOSTAL, «Gadamer's Relation to Heidegger and Phenomenology. Robert Dostal (ed.), The Cambridge Companion to Gadamer. Cambridge: Cambridge UP, pp. 247-266, 2002.

G. FIGAL, For a Philosophy of Freedom and Strife. Politics, Aesthetics, Metaphysics. (Orig. Für eine Philosophie von Freibeit und Streit, 1994). Trad. Wayne Klein. Nueva York: State University of New York Press, 1998.

G. FIGAL, «Philosophische Hermeneutik-hermeneutische Philosophie. Ein Problemaufriss". - Hermeneutische Wege. Hans-Georg Gadamer zum Hundersten. Ed. Günter Figal, Jean Grondin \& Dennis J. Schmidt. Tubinga: Mohr (Paul Siebeck), pp. 335$344,2000$.

M. FoucaulT, "What Is Enlightenment?» - Rabinow, Paul (ed.), The Foucault Reader. Nueva York: Pantheon Books, pp. 32-50, 1984.

M. FRANK, What is Neostructuralism? (Was ist Neostrukturalismus? 1984). Mineápolis: University of Minnesota Press, 1989.

H.-G. GadAmer, Philosophical Hermeneutics. Trad. y ed. David E. Linge. Berkeley: University of California Press, 1977.

H.-G. GADAMER, Reason in the Age of Science. Trad. F. G. Lawrence. Cambridge: MIT Press, 1981.

H.-G. GADAMER, «The Hermeneutics of Suspicion». - Gary Shapiro \& Alan Sica (eds.), Hermeneutics: Questions and Prospects. Amherst: The University of Massachusetts Press, pp. 54-65, 1984.

H.-G. GaDAmER, Reason in the Age of Science. Trad. Frederick G. Lawrence. Cambridge: MIT Press, 1986.

H.-G. GADAMER, «The Problem of Historical Consciousness». - Paul Rabinow \& William Sullivan (eds.), Interpretive Social Science. A Second Look. Berkeley: University of California Press, pp. 82-140, 1987.

H.-G. GADAMER, Gesammelte Werke. Band 1: Hermeneutik I. Wabrheit und Methode: Grundzigge einer philosophischen Hermeneutik (1960). Tubinga: Mohr (Paul Siebeck). = GW1, 1990 .

H.-G. GADAMER, Gesammelte Werke. Band 2: Hermeneutik II. Wabrheit und Methode. Ergänzungen, Register. Tubinga: Mohr (Paul Siebeck). = GW2, 1993. 
H.-G. GaDAMER, Gesammelte Werke. Band 10: Hermeneutik im Rückblick. Tubinga: Mohr (Paul Siebeck). = GW10, 1995.

H.-G. GaDAMER, Truth and Method (Wahrheit und Methode, 1960). Segunda ed., revisada. Trad. Joel Weinsheimer \& Donald G. Marshall. Nueva York: Continuum. = TM, 1997.

H.-G. GaDamer, "Reflections on my Philosophical Journey». - Lewis Hahn (ed.), The Philosophy of Hans-Georg Gadamer. Peru (Illinois): Open Court, pp. 3-57, 1997 a.

H.-G. GadAMER, «Reply to David Detmer». - Lewis Hahn (ed.), The Philosophy of HansGeorg Gadamer. Peru (Illinois): Open Court, p. 287, $1997 \mathrm{~b}$.

H.-G. GadAMER, "Reply to G. B. Madison». - Lewis Hahn (ed.), The Philosophy of HansGeorg Gadamer. Peru (Illinois): Open Court, pp. 366-367, 1997c.

H.-G. GADAMER, "Who Am I and Who Are You?" and other essays. Trad. y ed. Richard Heinemann \& Bruce Krajewski. Nueva York: State University of New York Press, 1997 d.

A. GIDDENS, New Rules of Sociological Method: A Positive Critique of Interpretative Sociologies. Londres: Hutchinson, 1976.

A. GiddenS, «Hermeneutics and Social Theory». - Gary Shapiro \& Alan Sica (eds.), Hermeneutics. Questions and Prospects. Amherst: The University of Massachusetts Press, pp. 115-230, 1984.

H. HABERMAS, «Der Universalitätsanspruch der Hermeneutik». - Jürgen Habermas, Dieter Henrich \& Niklas Luhmann (eds.), Hermeneutik und Ideologiekritik. Fráncfort del Meno: Suhrkamp, pp. 120-159, 1971.

H. HABERMas, The Theory of Communicative Action. Vol 1. Reason and the Rationalization of Society (Theorie des Kommunikativen Handelns. Band I: Handlungsrationalität und gesellschaftliche Rationalisierung, 1981). Boston: Beacon Press, 1984.

H. HABERMAS, "A Review of Gadamer's Truth and Method" (1967). - Brice R. Wachterhauser (ed.), Hermeneutics and Modern Philosophy. Nueva York: State University of New York Press, pp. 243-276, 1986a.

H. Habermas, "Life-forms, Morality and the Task of the Philosopher». - Peter Dews (ed.), Habermas. Autonomy \& Solidarity. Londres: Verso, pp. 191-216, 1986b, .

H. HABERMAS, “Wie ist nach dem Historismus noch Metaphysik möglich?» - „Sein, das verstanden werden kann, ist Sprache». Hommage an Hans-Georg Gadamer. Fráncfort del Meno: Suhrkamp, pp. 89-99, 2001. 
M. Heidegger, (1927), Sein und Zeit. Tubinga: Max Niemeyer Verlag, 1953.

H. R. JAUsS, Question and Answer. Forms of Dialogic Understanding. Ed. y trad. de Michael Hays. Mineápolis: University of Minnesota Press, 1989.

D. Jervolino, "The Depth and Breadth of Paul Ricœur's Philosophy». - Lewis Edwin Hahn (ed.), The Philosophy of Paul Ricoeur. Chicago: Open Court, pp. 533-543, 1995.

I. KANT, «Beantwortung der Frage: Was ist Aufklärung?» (1783) - I. Kant, Werke. Band $V I$. Ed. Wilhelm Weischedel. Darmstadt: Wissenschaftliche Buchgesellschaft, pp. 53-61, 1983.

P. KEMP, «Ethics and Narrativity». - Lewis Hahn (ed.), The Philosophy of Paul Ricour. Peru (Illinois): Open Court, pp. 371-394, 1995.

T. KuHN, "The Natural and the Human Sciences». - David Hiley, James Bohman \& Richard Shusterman (eds.), The Interpretive Turn. Philosophy, Science, Culture. Ithaca: Cornell UP, 1991.

G. B. MADISON, "Ricœur and the hermeneutics of the subject». - Lewis Hahn (ed.), The Philosophy of Paul Ricaur. Peru (Illinois): Open Court, pp. 75-92, 1995.

G. B. MADISON, "Hermeneutics' Claim to Universality». - Lewis Hahn (ed.), The Philosophy of Hans-Georg Gadamer. Peru (Illinois): Open Court, pp. 349-365, 1997.

G. B. MADISON, The Politics of Postmodernity. Essays in Applied Hermeneutics. Dordrecht: Kluwer, 2001.

T. MCCARTHY, The Critical Theory of Jürgen Habermas. Cambridge: Polity Press, 1984.

P. RICGUR, «L'histoire comme récit et comme pratique. Entretien avec Paul Ricœur». Esprit, n. 54, pp. 155-165, 1981.

P. RicceuR, Time and narrative. Vol 3. (Temps et récit III, 1985). Trad. Kathleen Blamey \& David Pellauer. Chicago: University of Chicago Press, 1990.

P. RICcEUR, From text to action. Essays in hermeneutics II. Trad. Kathleen Blamey \& John B. Thompson. Evanston: Northwestern UP, 1991.

P. RICceur, Oneself as Another. (Soi-même comme un autre, 1990). Trad. Kathleen Blamey. Chicago: Chicago UP, 1994.

C. TAYLOR, "The Dialogical Self». - David Hiley, James Bohman \& Richard Shusterman (eds.), The Interpretive Turn. Philosophy, Science, Culture. Ithaca: Cornell UP, pp. 304-314, 1991. 
C. TAYLOR, "The Validity of Transcendental Arguments". - Charles Taylor, Philosophical Arguments. Cambridge: Harvard UP, pp. 20-33, 1995.

C. TAYLOR, "Gadamer on the Human Sciences". - Robert Dostal (ed.), The Cambridge Companion to Gadamer. Cambridge: Cambridge UP, pp. 126-142, 2002a.

C. TAYLOR, "Understanding the other: A Gadamerian View on the Conceprual Schemes». - Jeff Malpas, Ulrich Arnswald \& Jens Kertscher (eds.), Gadamer's Century. Essays in Honor of Hans-Georg Gadamer. Cambridge: MIT Press, pp. 279-297, 2002b.

G. VATTIMO, 1988, «Hermeneutics as Koine». Theory, Culture of Society, vol. 5 (2-3), pp. 399-408, 1988.

G. WarnKe, Gadamer. Hermeneutics, Tradition and Reason. Cambridge: Polity Press, 1987.

G. WARNKE, "Hermeneutics, Ethics, and Politics». - Robert Dostal (ed.), The Cambridge Companion to Gadamer. Cambridge: Cambridge UP, pp. 79-101,. 2002a.

G. WARNKE, «Social Identity as Interpretation». - Jeff Malpas, Ulrich Arnswald \& Jens Kertscher (eds.), Gadamer's Century. Essays in Honor of Hans-Georg Gadamer. Cambridge: MIT Press, pp. 307-329, 2002 b.

J. WeInSHeImer, Philosophical Hermeneutics and Literary Theory. New Haven \& Londres: Yale UP, 1991. 